\title{
Rock Casting Slice Image Based 3D Digital Core Reconstruction
}

\author{
$\mathrm{Na} \mathrm{Liu}{ }^{1,2}$ \\ ${ }^{1}$ National Engineering Laboratory for Exploration \\ and Development of Low-Permeability Oil \& Gas \\ Fields \\ ${ }^{1}$ NDRC \\ ${ }^{1}$ Xi'an, China \\ ${ }^{2}$ Research Institute of Exploration and \\ Development \\ ${ }^{2}$ PetroChina Changqing Oilfield Company, CNPC \\ ${ }^{2}$ Xi'an, China \\ e-mail: liun1_cq@petrochina.com.cn
}

\section{Wei Liu ${ }^{1,2}$}

${ }^{1}$ National Engineering Laboratory for Exploration and Development of Low-Permeability Oil \& Gas

$$
\begin{gathered}
\text { Fields } \\
{ }^{1} \text { NDRC } \\
{ }^{1} \text { Xi'an, China }
\end{gathered}
$$

\author{
${ }^{2}$ Research Institute of Drilling \& Production \\ Engineering Technology \\ ${ }^{2}$ Chuanqing Drilling Engineering Company \\ Limited, CNPC \\ ${ }^{2} \mathrm{Xi}$ 'an, China \\ e-mail:gcy_liuw@cnpc.com.cn
}

\author{
Guojian Cheng \\ School of Computer Science \\ Xi'an Shiyou University \\ Xi'an, China \\ e-mail: gjcheng@xsyu.edu.cn
}

\author{
Xinjian Qiang * \\ School of Computer Science \\ Xi'an Shiyou University \\ Xi'an, China \\ e-mail: qiangxj@xsyu.edu.cn
}

\begin{abstract}
For the reason of high costing on 3D core reconstruction by using CT scanning method, a new method named 3D digital core reconstruction based on rock casting slice images under optical microscope is proposed in this paper. Firstly, the collected casting slice images are processed to acquire the reconstructed image templates, and then multiple-point geostatistics is applied for feature extracting on these image templates and 3D reconstructing on the digital core. The core samples used in the experiment are taken from Sulige region in Ordos basin. The experiment outcome shows that the core aperture distributions and the real value have the same trend and the relative error of porosity is lower than $8 \%$. The above researches show that the rock casting slice image based 3D Digital Core Reconstruction methodology is effective.
\end{abstract}

Keywords-Casting Slice Image; Digital Core; 3D Reconstruction; Image Processing; Multiple- Point Geostatistics

\section{INTRODUCTION}

The 3D reconstructing on digital core is aiming at achieving the pore network modeling (PNM) of rock. PNM was proposed as an equivalent model for describing the complex pore space structure of rock. The accurate and detailed microstructure cannot be estimated by only using $2 \mathrm{D}$ rock casting slice image, so the determination method must be figured out based on 3D models. Generally, the traditional manually modeling method is tedious, timeconsuming and low efficiency, not to mention the modeling of the complicated pore structure distribution in the rock samples, therefore, it is very necessary to develop a high efficient automation technology for 3D reconstruction on digital core, which can help researchers to reduce the design cycle, cost and finally improve the economic benefits [1]. For example, the applying of 3D reconstruction on digital core in the reservoir physical property experiment can largely reduce the experiment time-consuming compared to the traditional physical experiment and it can improve the timeliness of the experimental outcomes, especially the core samples can be reused as it will not be damaged in the experiment.

In the research of digital core 3D reconstruction Jun Yao, Xiucai Zhao, Guo Tao etc. in China University of Petroleum have done a lots of work including systematic research on constructing the theory and methods to establish artificial digital core, sandstone and carbonate with CT scan method, simulated annealing method and process simulation method, grain-size composition curve based on core slice or core data, and finally they evaluated the characteristics and seepage simulation properties of the digital core via micro pore space structure analysis and the lattice Boltzmann method[2,3,4]. Detang Lu, Feng Huang, and Ting Zhang etc. in University of Science and Technology of China studied 3D reconstruction of core pore network by studying the core pore space structure characteristics of 2D images acquired by scanning electron microscopy, moreover, they discussed several 3D model reconstruction methods based on $2 \mathrm{D}$ image statistical information, especially the multiple-point geostatistics in the application of 3D reconstruction of digital core[5,6,7]. Multipoint statistical research had been successfully applied to the 3D reconstruction of digital core by 
Tuanfeng Zhang in American academy of schlumberger Doll[8]. Professor Martin Blunt in Imperial college and his modeling team of reservoir pore, which represents the highest level over the world, worked related to the description of the digital core imaging technology of nanometer scale as well as the different digital technology in simulating single-phase flow and multiphase flow migration, he also summarized the limitation and challenges in different quantity level of imaging and in simulating fluid migration in the core pore space and determination of moisture[9].

In the above research, the digital core is reconstructed mainly based on the CT scanning method or local 2D image. Unfortunately, without using rock casting slices, which is an important part in the study of petrology fundamental research researchers can understand the type of reservoir rock, mineral composition, porosity and pore topology through studying the casting slices under optical microscope and researchers can quickly and accurately obtain rock samples parameters through analyzing and processing the high resolution casting slice images with computer technology. The feature information in $2 \mathrm{D}$ images can be extended to $3 \mathrm{D}$ space to simulate rock micro $3 \mathrm{D}$ structure and in the whole process only a small amount of casting slices of rock samples are needed, therefore, the casting slices can be obtained conveniently and economically which possesses a lot of advantages. At this point, 3D reconstruction of digital core based on casting slices under optical microscope is proposed in this paper. Firstly, the training image templates for 3D reconstruction is obtained by processing and analyzing the casting slice images, and then multiple-point geostatistics is applied to extract image template features and digital core 3D reconstruction researching, finally, the physical parameters in the $3 \mathrm{D}$ reconstruction model are compared with them in the practical rock samples, so as to discuss the effectiveness of $3 \mathrm{D}$ reconstruction of digital core based on casting slice images.

\section{CASTING SLICE IMAGE}

Most researches on 3D reconstruction of digital core use CT scanning method, namely the X-ray stereoscopic imaging method. The 3D reconstruction requires the following three steps, firstly, preprocessing the rock samples and obtain projection data through CT scanning experiment; secondly, using the corresponding image reconstruction method to analyze the data obtained in the first step, thirdly, segmenting the pore space and the rock skeleton in the grayscale image so as to establish the digital core. CT scan imaging is based on the principle of $\mathrm{X}$-ray intensity attenuation, so that researchers can measure the X-ray signal strength after penetrating rock section and then calculate the absorption data corresponding to the space rock fault location, accordingly, researchers can obtain the structure information of the rock samples section [10].

It is different from CT scanning method for 3D reconstruction of digital core, this paper uses casting slice images of the rock samples, which can be obtained conveniently and economically, therefore it has more advantages. The rock slices in this paper are taken from the vertical direction of the bedding rock by cutting a small piece of rock slice; it is grinded into $0.03 \mathrm{~mm}$ to stick on the glass slide. The rock slices are observed and identified under polarizing microscope so as to determine the type of rock and structure parameters. The casting slices is one types of rock slices. Colored resin or liquid rubber (red or blue) is infused into the pore space of rock under vacuum, it will become consolidated under a certain temperature and pressure and then it will be grinded into rock slice to observe the size of pore, throat and the interconnected, assembled two-dimensional spatial structures under polarized light microscope.

The rock slice image in this paper is from Sulige region in Ordos basin, all of which are casting slices and the pore space is filled with red colloid. The main gas-bearing sections in this region is Box 8 Paragraph of the Lower Shihezi Formation in Lower Permian and sandstone reservoir in fluvial facies - delta of Shan 1 section in Shanxi Formation, among them the Box8 Paragraph is mainly comprised of coarse sandstone, glutenite and medium-coarse sandstone, Shan 1 reservoir paragraph is mainly comprised of quartz sandstone. Sedimentary microfacies in gas reservoir are mainly in the river beach, with big particle size, it mostly include gravel coarse sand, sandstone. Gas reservoir mainly develops intergranular dissolved pore, intragranular dissolved pore, and the average porosity is greater than $5 \%$, permeability is greater than $0.15 \times 10-3 \mu \mathrm{m} 2$. According to the correlation analysis of porosity and permeability of the core sample, in the reservoir of Sulige area, the permeability and porosity show obvious positive correlation, which means that the changes of permeability is influenced by porosity development degree[11]. Therefore, casting slice images are applied to 3D reconstruction of digital core, base on that, the 3D reconstruction of digital core model is combined with percolation theory for subsequent research on microscopic percolation simulation.

\section{METHOD}

3D reconstruction method of digital core based on casting slices under optical microscope is divided into two parts, image processing and 3D reconstruction using multiple-point geostatistics. Firstly, the training image templates for 3D reconstruction is obtained by processing and analyzing the casting slice images, then multiple-point geostatistics is applied to extract image template features in order to take research on 3D reconstruction of digital core.

\section{Image Processing}

The purpose of the image processing is to separate from the pore and framework of rock, mainly including color space conversion, color component representation and image segmentation and morphological processing[12]. Color space: In the casting slices image research, all images acquired are in JPG format, which are processed by MATLAB and showed in the RGB color space. RGB (red, green, blue) color space is one of the most common used color Model, but it is not the only color space. Displaying on computers often uses RGB color space, and red, green and blue, are used as $\mathrm{X}, \mathrm{Y}$, and $\mathrm{Z}$ axes respectively. However, RGB color space can be transformed into another color space, such as $\mathrm{YCbCr}, \mathrm{Y}$ is the luminance component, $\mathrm{Cb}$ is the blue component, and 
$\mathrm{Cr}$ is the red component. Among them, eyes are most sensitive to the luminance in images or video, so selecting the component of color in image processing can reduce the impact of luminance and image quality. After comparing the distribution of the rock skeleton and pore showed in casting slice images in multiple color space and component, finally $\mathrm{Cr}$ component in $\mathrm{YCbCr}$ color space is selected as the follow-up in the research of image segmentation, morphological object .

Image segmentation: The casting thin sections of image binarization processing, which is a segmentation research for rock skeleton and pore that is based on $\mathrm{Cr}$ image component which gets from the study of image color space. Image binarization processing which sets the points' gray level on the image to 0 or 255 , this can make the entire image appear with a distinct black and white effect. This binary image, comes from segmentation after get threshold value of 256 brightness levels of gray image, this kind of image can keep the original geometric features on the global and local. Threshold segmentation of the image processing technology which has simple operation, small amount of calculation and stable performance is usually one of the common image segmentation method, which can be obtained through this method, setting the original image to $f(x, y)$, using the characteristic value of $\mathrm{T}$ which can be found according to certain rules in $\mathrm{f}(\mathrm{x}, \mathrm{y})$ to divide the image into two parts. The casting thin sections of image binarization results are got from the $\mathrm{Cr}$ components by automatic threshold segmentation, white among them is pore and black is rock skeleton.

Morphological processing: Corresponding noise will be produced due to image threshold segmentation, which affects image quality. Aiming at solving these problems researchers produce the casting thin section image enhancement and morphological processing. In image enhancement, the main application of median filtering noise processing technology is applied to Fig. 3, the median filter in filtering out the noise at the same time will filter out small throat between rock skeleton particles, so do some morphology processing work at the same time, extract the image skeleton line, that is the throat of rock pore space. Finally, the image of the resulting from the median filter will be fused with skeleton line image, then the training images that digital core $3 \mathrm{D}$ reconstruction are needed will be created.

In the fusion image which gets from median filter and morphological transformation, porosity and rock skeleton get better segmentation, and other important microcosmic structure information, like throat, is preserved. For digital core image $3 \mathrm{D}$ reconstruction of the casting thin sections are in accordance with the color space conversion, component extraction, image segmentation, image enhancement and morphological transform image fusion after a process for processing.

\section{Multiple-point geostatistics}

The concept of Multi-point simulation is put forward by Guardiano and Srivastava in 1993, the spatial patterns of training images instead of the two structures that are produced by the traditional two-point in geostatistics variogram model. Its essence is the MPS trying to match a set of complete data; the match is not looking for one at a time benchmark, but a match which multiple data values at a time benchmark together that is 'multipoint'. Using Multipoit geostatistics method which combines the parameters of the pixel method is easy to control with copy model based on the target method as well as the good efficiency in time and space for 3D reconstruction of porous media. MPS method process is extracted the information of multipoint geostatistics from the training image and form some refactoring mode, then copies these patterns to reconstruct image. For reservoir structure reconstruction, it needs geological image as training images; for core pore structure of 3D reconstruction, it can use the micro scale high precision casting thin sections of the image astraining images.

Because the speed of original multi-point statistical algorithm which created by Guardiano is slow, this algorithm requires to scan the entire training images again for each multi-point event in every simulation nodes. In 2000, Strebelle puts forward practical multipoint statistical concepts of single normal equation simulation (SNESIM). In SNESIM algorithm, scanning the training image only once, the proportion of all conditions in training will be scanned images are stored in the search tree data structure, so that who can carries on the retrieval effectively. SNESIM algorithm contains two main parts: the proportion of all training images that are stored in search tree structure; query and call the proportion of parts to extract simulation value. The total number of nodes in search template is the evaluation standard of multi-point statistical, the greater number researchers get, the final simulation results will be better. However, as the total number of nodes increases, the consumption of memory will increase rapidly. Therefore, for most of the 3D simulation, it needs to choose a moderately total number of nodes, and the effect will be better.

The steps of the SNESIM algorithm:

- Step 1: define a search template.

- Step 2: aiming at search template to create search tree.

- Step 3: the hard data reset to the nearest simulation grid nodes, and be fixed in the simulation.

- $\quad$ Step 4: define a random path access to the storage unit which be waited for simulating.

- Step 5: iterate through every storage unit of this path.

- Step 6: use search template to define the condition data events.

- Step 7: to regain conditional probability distribution function from the search tree.

- Step 8: put the simulated values which get from conditional probability loaded into the data setup.

- $\quad$ Step 9: end of cycle.

Based on the above multiple-point geostatistics, this paper uses SNESIM algorithm for digital core of the casting thin sections image three dimensional reconstructions. By the results of image processing, use the search template to get the statistical characteristics of core pore structure, and then establish the search tree of conditional probability distribution function through this; 
simulate the assignment on all units of the 3D model according to algorithm steps to complete the 3D reconstruction of digital core.

\section{RECONSTRUCTION EXAMPLES}

In order to test the feasibility and reliability of the method, lotus core samples were selected from 3771.26 meters deep in Su 279 well, 3795.31 meters deep in Lian 47 well and 1939.65 meters deep in Shen 41 well from

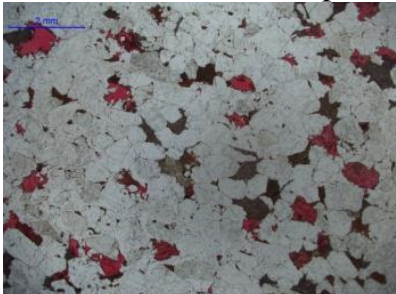

(a) $\mathrm{Su} 279$

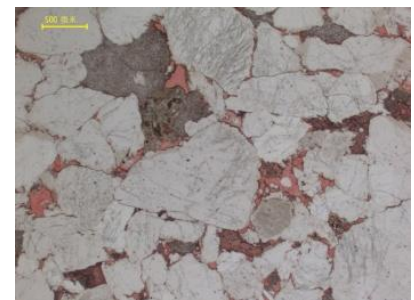

(b) Lian 47
Ordos basin Sulige. Core slice images were collected under optical microscopes and three-dimensional reconstruction was developed for digital core. Because three core samples were collected from different geological areas, they were with inconsistent petrological characteristics as well as different heterogeneity and pore structures. Fig. 1 showed the images of casting slices in three groups.

Figure 1. Casting slice images for testing.

The images of casting slices in three groups shown in Fig. 1 were modified sequentially under the process according to above methods and with following order: color space conversion, component extraction, image segmentation, image enhancement and image fusion morphological transformation. Binarization images after processing for casting slices in three groups were shown in Fig. 2 and these rock matrix porosity binarization images would be used for three-dimensional reconstruction of the digital lotus cores.

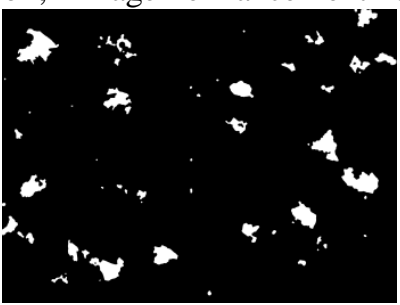

(a) $\mathrm{Su} 279$

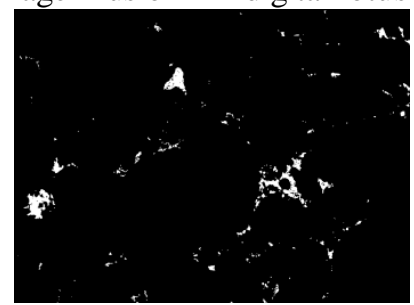

(b) Lian 47

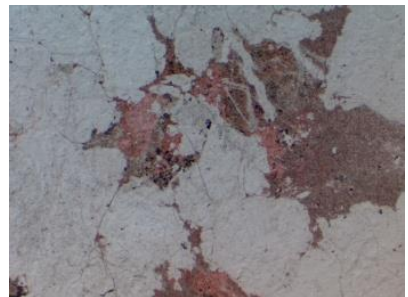

(c) Shen 41

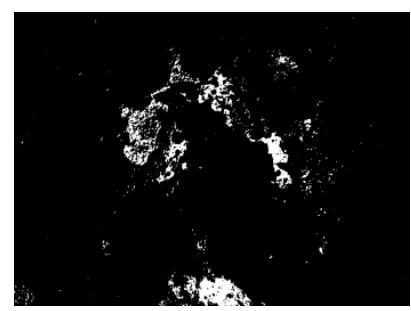

(c) Shen 41

Figure 2. Binarization images after processing

The 3 groups of binarization images in Fig. 2 were used as the training images and reconstructed separately using SNESIM algorithm. In the algorithm, three points neighborhood search template were chosen and used for traversing the training images above to extract image features and reconstruction mode. Based on this, a searching tree with conditional probability distribution function was established according to statistical parameters. Then three-dimensional pore structures of digital cores were reconstructed according to the algorithm process. Fig. 3 showed a few binary reconstruction results randomly selected from the reconstruction model.

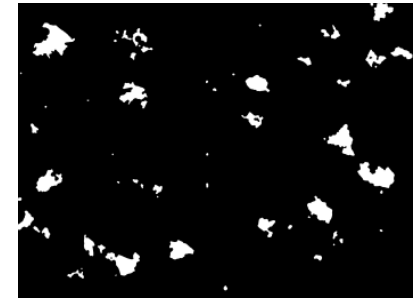

(a) Su 279

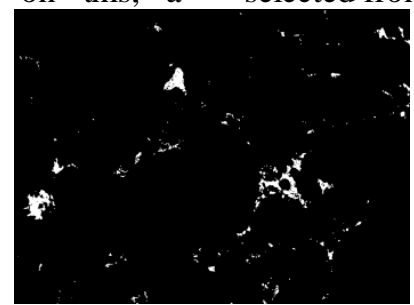

(b) Lian 47

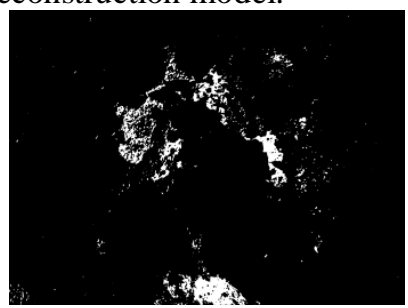

(c) Shen 41

Figure 3. Reconstruction results

By comparing Fig. 2 \& Fig. 3, researchers can see each of the 3 groups of reconstruction images were showing different features of the original training images. To quantitatively evaluate the performances of the reconstruction results, researchers used porosity as the reconstruction evaluation index and the results of the comparison were shown in Table I. Based on Table I, researchers can see reconstruction results for all 3 groups of cores casting slices were with relative errors less than $8 \%$. Among them, Lian 47 well showed the lowest relative error which is $5.0 \%$ and the mean of the relative errors was $6.1 \%$ so researchers can claim that the three-dimensional reconstruction for digital core based on cores casting slices were overall accurate. Meanwhile, in order to demonstrate the performance of the reconstruction for digital core in three-dimensional space, Fig. 2(a) was selected as the training image to build the $3 \mathrm{D}$ reconstruction model of digital core, which was shown in Fig. 4.

In Fig. 4(a), the gray represented the rock matrix and the red represented the pore. Pore carving body was shown 
in Fig. 4(b) after removing the rock matrix to improve the demonstration of the digital core distribution. As can be seen from the figure, the reconstructed digital core offered a good exhibition in 3D space on the pore structure characteristics and continuity of the rock samples from Fig. 2(a). Also it showed similar pattern features when compared to the training images both horizontally and vertically.

TABLE I. COMPARISON OF ORIGINAL IMAGE AND RECONSTRUCTED IMAGE

\begin{tabular}{|c|c|c|c|}
\hline Sample & Su 279 & Lian 47 & Shen 41 \\
\hline porosity/ \% & 4.1 & 2.0 & 3.3 \\
\hline Reconstruction porosity/ \% & 4.4 & 2.1 & 3.1 \\
\hline relative error/ \% & 7.3 & 5.0 & 6.0 \\
\hline
\end{tabular}

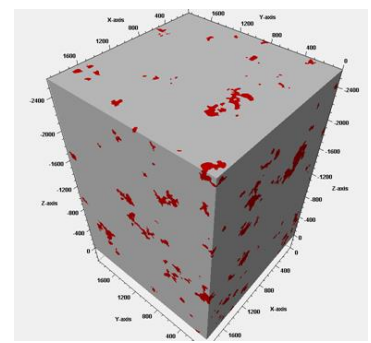

(a) $\mathrm{Su} 279$

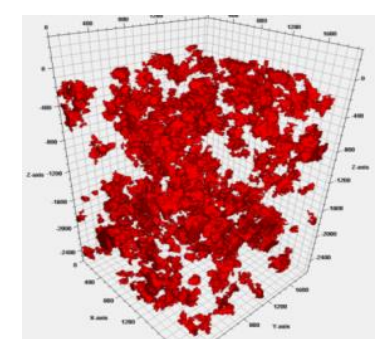

(b) Lian 47
Figure 4. Reconstruction results

In summary, based on the comparison porosity and pore size distribution, the reconstructed digital core and the original casting slices are quite similar, so the feasibility and reliability of the algorithm were verified. Therefore, this multi-point geostatistical digital core algorithm based on the core slices can extract the pore structures from the slice images with conditional probability searching tree to make sure the reconstruction results are consistent with the pore structural features from the original images. Also, this method can remain the randomness, which is in line with the nature of the spatial distribution of the underground rock pore structure.

\section{CONCLUSIONS}

This article came up with a digital core 3D reconstruction method based on the casting slice images obtained from optical microscopes. Despite the expensive costs of the digital core $3 \mathrm{D}$ reconstruction methods based on the CT scan, it is convenient and economical to study the casting slice images of the rock samples. This method can obtain binary images as the training templates by processing color space conversion, component representation, image segmentation, image enhancement and morphological modification to the casting slice images. Then template image feature extraction and threedimensional reconstruction of the digital core are implemented using multi-point geostatistics. The experiment used rock samples from Su 279, Lian 47 and Shen 41 in Ordos basin Sulige as a target to test the reconstruction. According to the results, the digital core pore size distribution from the reconstruction showed the same trend compared to the real values and the porosity was with relative errors less than $8 \%$. The experiment verified the feasibility and reliability of the digital core 3D reconstruction method based on the casting slice images. Meanwhile, the research was still with insufficient parts. For example, when the pixel density of the casting slice images was too large, the time cost of 3D reconstruction would be also large. In the future study, researchers will focus on improving the accuracy and speed of the $3 \mathrm{D}$ reconstruction.

\section{ACKNOWLEDGMENT}

The research work is supported by National Key S\&T Special Projects of China -- Major oil \& gas field and coal bed methane exploitation (2011ZX05044, 2011ZX05001004). It is also supported by Industrial S\&T research project of Shaanxi province in China -- Microscopic scale intelligent characterization of tight sandstone reservoir in Ordos basin (2015GY104) .

\section{REFERENCES}

[1] Tao Jun, Yao Jun,and Zhao scholar,. "Use IRIS Explorer data visualization software for digital core porosity level visualization,". Oil and Gas Technology, vol. 28(5), pp. 51-53, 2007.

[2] Zhao Xiucai, Yao Jun, and Fang Kerong, "The new method of Reasonable split core microstructure image," China University of Petroleum (Natural Science), vol. 33(1), pp. 64-67, 2009.

[3] Zhao Xiucai, "Research of digital core and pore network model reconstruction method," Dongying: China University of Petroleum (East China), 2009.

[4] Zhu Yihua, TAO Guo, Fang Wei .Application of digital image processing technology in core modeling $[\mathrm{J}]$. Oil and Gas Technology, vol. 29(5), pp. 54-57, 2007.

[5] Zhang Ting, LU De-tang, and Li Daolun, "A three-dimensional reconstruction of porous media based on two-dimensional images and multi-point statistical methods," University of Science and Technology of China, vol. 40(3) , pp. 271-277, 2010.

[6] Huang Feng, "Research of three-dimensional reconstruction of porous media model," Hefei: University of Science and Technology of China, 2007.

[7] Hang Ting, "Porous media reconstruction method and realization based on Porous geostatistics," Hefei: University of Science and Technology of China, 2009.

[8] Zhang T F, Switzer P, and Journel A G. "Filter-based classification of training image patterns for spatial simulation," Mathematical Geology, vol. 38(1), pp. 63-80, 2006.

[9] Okabe H, Blunt M J. Pore space reconstruction using multiplepoint statistics $[\mathrm{J}]$. Journal of Petroleum Science and Engineering, vol. 46(1), pp. 121-137, 2005.

[10] Desbois G, Urai J L, Kukla P A, et al. "High-resolution 3D fabric and porosity model in a tight gas sandstone reservoir: a new approach to investigate microstructures from $\mathrm{mm}$-to nm-scale combining argon beam cross-sectioning and SEM imaging," Journal of Petroleum Science and Engineering, vol. 78(2), pp. 243-257, 2011.

[11] Zhu Xiaomin, Sun Chao, LIU Cheng-lin, etc. Diagenesis Sulige gas field in the Ordos Basin and simulation [J]. Chinese Geological, vol. 34(2) , pp. 276-282, 2007.

[12] Zhang Zheng, Ni Hongxia, Yuan Chunmiao, proficient in Matlab and other digital image processing and recognition [M], Beijing: People's Posts and Telecommunications Press, pp. 197-272, 2013.

[13] Remy N, Boucher A, Wu J. Applied geostatistics with SGeMS: A user's guide[M]. Cambridge University Press, pp. 168-191, 2009.

[14] Wu Shenghe, Li Wenke, "Multi-point geostatistics - Theory, Applications and Prospects," Palaeogeography, vol. 7(1), pp. 137144, 2005. 